\title{
Egg bank investment
}

\section{Winfried Lampert}

PlANKTONIC organisms in lakes are usually small and short-lived, responding quickly to changes in the environment and showing typical seasonal successions ${ }^{1}$. But some manage cunningly to increase their lifespan by assuming a dormant stage that settles into the sediment, where they wait for favourable conditions to establish new populations. These resting stages can survive impressive periods of diapause: for example, akinetes of the cyanobacterium Anabaena have survived for 60 years ${ }^{2}$, and zooplankton eggs as old as 35 years are still viable. But now, writing in Ecology ${ }^{3}$, Hairston and his co-workers report a new world record: from the sediment of a small freshwater lake in Rhode Island, they have hatched resting eggs of the copepod Diaptomus sanguineus that are probably 330 years old.

Diaptomus sanguineus is a small (1 mm body length) calanoid copepod, distributed throughout most of North America, that lives in permanent lakes and ponds as well as in temporary ponds. In winter it produces eggs that hatch quickly and develop immediately into nauplius larvae, but it switches to diapausing resting eggs in spring to escape drying out in temporary ponds during the summer and to avoid predation by planktivorous fish in permanent water bodies ${ }^{4}$. The diapausing eggs are about $100 \mu \mathrm{m}$ in diameter. They possess a thick chitinous shell that makes them resistant to desiccation and allows them to survive passage through a predator's gut.

Hairston et al. selected two small lakes for their study. The larger one is permanent, with a surface area of 2.4 hectares and a maximum depth of 4.0 metres. The smaller one ( 0.15 hectares, 1.5 metres) dries out occasionally - the last time was in 1981. Scuba divers took sediment cores which were sliced into 1-cm layers. Diaptomus resting eggs were isolated from each layer, then counted and hatched in an incubator. Sediment slices from a parallel core were dated by ${ }^{210} \mathrm{~Pb}$ activity. This allowed the calculation of sedimentation rates $(0.5-0.8 \mathrm{~mm}$ per year) and indirect dating of the eggs found at different sediment depths.

Although egg numbers declined with sediment depth (mortality rate was about 1.5 per cent per year), some eggs were still present $31 \mathrm{~cm}$ below the surface in the larger lake and were estimated to be about 400 years old. The mean egg age in the lake was about 70 years. As dead eggs decay quickly, it was fair to assume that these eggs were still viable, although the oldest eggs that could be hatched were 330 years old. Only a fraction of the isolated eggs could be hatched from all depths, but this is not unusual as the optimum environmental cue to terminate diapause and induce development of the embryo is

slowly recolonized by dispersal of organisms from unaffected lakes. The egg bank functions as a time-dispersal system that allows immediate recolonization.

This idea has been further developed with respect to the possible consequences of global climate change on lake biota ${ }^{5}$. A change in mean temperature could well be accompanied by a change in its variance $^{6}$. Zooplankton that cannot physiologically tolerate a series of extreme years can still survive by investing in an egg bank. Hence the egg bank may become increasingly important for species diversity and the structure of lake foodwebs, which has an indirect effect on lake productivity and nutrient dynamics.

Seed-bank theory predicts that fluctuations in recruitment should lead to the evolution of prolonged diapause ${ }^{7}$. There is not enough known yet about egg banks to decide whether they are adaptive or just an artefact. Will the eggs buried in the sediment ever hatch in nature? Hairston et al. think that this is possible if the sediment is perturbed and old eggs are moved to the surface where they experience the proper environmental cues. There are many possibilities for sediment disturbance from feeding fish to falling trees to limnologists' anchors.

One striking example of how sediment disturbance can affect lake plankton communities was reported when the top sediment layers of Lake Trummen in Sweden were removed for restoration purposes ${ }^{8}$. This

still unknown (probably a combination of oxygen, light and temperature).

What is the significance of this remarkable result? Diapausing egg densities at the sediment surface were about $5 \times 10^{4}$ $\mathrm{m}^{-2}$ and the total number of eggs waiting to hatch in the larger of the two lakes was about $6.5 \times 10^{9}$, which is 18 times greater than the annual mean maximum population size of adult copepods in the open water. These eggs represent an egg 'bank', analogous to the seed banks of many terrestrial plants. Simultaneous hatching of larvae from eggs produced in different years produces a large overlap between generations, which slows the rate of microevolution ${ }^{4}$. It also decreases the rate of elimination of a species from a system when conditions for active stages become unfavourable, and increases the rate of recolonization of a water body when conditions improve. With non-overlapping generations, a single harsh year with failure of reproduction will result in the elimination of the species from the lake or even a given region. The system may be mechanical disturbance was followed by the appearance of large maxima of chrysophycean algae in the lake, probably from cysts that were liberated from lower sediment layers. The reminder of such findings, and Hairston and colleagues' dramatic discovery of survivors of environmental conditions from several hundred years ago, will stimulate numerous new studies in ecology and population genetics.

Winfried Lampert is in the Department of Physiological Ecology, Max Planck Institute for Limnology, Postfach 165, D-24302 Plön, Germany.

\footnotetext{
1. Sommer, U. et al. Arch. Hydrobiol. 106, 433-471 (1986).

2. Livingstone, D. \& Jaworski, G. H. M. Br. phycol. J. 15 357-364 (1980).

3. Hairston, N. G. Jr et al. Ecology 60, 1706-1711 (1995).

4. Hairston, N. G. Jr \& De Stasio, B. T. Jr Nature 336, 239-242 (1988)

5. Hairston, N. G. Jr Limnol. Oceanogr. (in the press) 6. Kerr, R. A. Science 268, 363-364 (1995).

7. Eliner, S. Theor. Populat Biol 28, 50-79 (1985)

8. Cronberg, G. Fol. Limnol. Scand. 18, 1-119 (1982).
} 